\title{
全氟辛基磺酸茂锆催化吲哚与羰基化合物反应
}

\author{
易卫国 ${ }^{a, b}$ 贾振永 ${ }^{b}$ 李宁波 ${ }^{b}$ 邱仁华 $b \quad$ 陈锦杨 ${ }^{b}$ 许新华 $*, b$

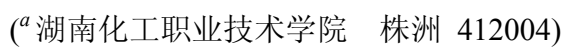 \\ ( ${ }^{b}$ 湖南大学化学化工学院 长沙 410082)
}

\begin{abstract}
摘要 在 $1.0 \mathrm{~mol} \%$ 二(全氟辛基磺酸)二茂锆存在下，吲哚与醛、酮在室温、四氢呋喃中反应 $10 \sim 25 \mathrm{~min}$, 生成二吲哚甲 烷衍生物，收率达 86\% 96\%. 催化剂二(全氟辛基磺酸)二茂锆能重复使用 6 次, 转化率仍有 $90 \%$; 反应不需要在严格 的无水条件下进行, 上述过程为二吲哚甲烷衍生物制备提供一条新的有效途径.

关键词 全氟辛基磺酸二茂锆; 催化; 合成; 二吲哚甲烷衍生物
\end{abstract}

\section{Zirconocene Bis(perfluorooctanesulfonate)s-Catalyzed the Reaction of Indoles and Carbonyl Compounds}

\author{
Yi, Weiguo $^{a, b} \quad$ Jia, Zhenyong ${ }^{b} \quad$ Li, Ningbo $^{b} \quad$ Qiu, Renhua $^{b}$ \\ Chen, Jinyang $^{b} \quad \mathrm{Xu}, \mathrm{Xinhua}^{*, b}$ \\ ( ${ }^{a}$ Hunan Chemical Industry Vocation Technology Institute, Zhuzhou 412004) \\ ( ${ }^{b}$ College of Chemistry Chemical Engineering, Hunan University, Changsha 410082)
}

\begin{abstract}
In the presence of $1.0 \mathrm{~mol} \%$ zirconocene bis(perfluorooctanesulfonate)s, indoles reacted with aldehyde/ketone at room temperature in tetrahydrofuran for $10 \sim 25 \mathrm{~min}$ to give diindolylmethane derivatives in $86 \% \sim 96 \%$ yields. The catalyst zirconocene bis(perfluorooctanesulfonate)s can be reused 6 times, and the conversion rate is still as high as $90 \%$. This reaction does not require strict anhydrous conditions. This procedure provides a new and efficient way for preparing diindolylmethane derivatives.
\end{abstract}

Keywords zirconocene bis(perfluorooctanesulfonate)s; catalysis; synthesis; diindolylmethane derivatives

路易斯酸化学在现代有机合成中一直扮演着重要 角色 ${ }^{[1 \sim 4]}$. 一般而言, 路易斯酸的酸性越强则它所具有 的反应活性越高, 但随着它的酸性增强, 对空气和水越 敏感，更容易水解. 这一矛盾一直未能解决. 第四族金 属为中心的阳离子茂合物是一类具有代表性的化合物, 对它们的研究已引起日益广泛的关注. 二茂金属三氟甲 磺酸配合物 $\mathrm{Cp}_{2} \mathrm{M}(\mathrm{OTf})_{2}\left(\mathrm{Cp}=\mathrm{C}_{5} \mathrm{H}_{5}, \mathrm{Tf}=\mathrm{CF}_{3} \mathrm{SO}_{2}\right)$ 已经 成功地应用到了各种碳一碳键的形成反应中 ${ }^{[5 \sim 8]}$. 然而 不足的是, 这类茂金属三氟甲基配合物在空气中, 容易 被水解, 所以它们必须在严格无水的条件下使用. 因此, 克服茂金属配合物易水解具有重要的应用价值.

Otera 小组 ${ }^{[9]}$ 报道, 全氟辛基磺酸锡配合物对空气 和水都相当稳定, 这与对应的具有高水解性的三氟甲基 磺酸锡形成鲜明对比. 这个发现表明, 长链全氟烷基磺
酸官能团能够克服阳离子茂金属配合物的水解不稳定 性. 因此, 我们制备了茂金属( $\mathrm{Ti}, \mathrm{Zr}, \mathrm{Hf})$ 全氟辛基磺酸 配合物和全氟苯基磺酸配合物, 并报道了它们在催化形 成碳一碳键反应中的应用 ${ }^{[10 \sim 15]}$.二吲哚甲烷衍生物具 有重要生理活性，其制备一般是采用 Lewis 酸如 $\mathrm{Dy}(\mathrm{OTf})_{3}{ }^{[16]}, \mathrm{ZrOCl}_{2}{ }^{[17]}, \mathrm{AlPW}_{12} \mathrm{O}_{40}{ }^{[18]}$ 等催化吲哚与羰基 化合物反应。但是, 这些方法存在一些不足, 如 $\mathrm{Dy}(\mathrm{OTf})_{3}$ 对水敏感, 不能回收和重复使用; 使用 $\mathrm{ZrOCl}_{2}$ 作催化剂需要使用硅胶作载体, 需要在加热条件下进 行, 且催化剂用量较大 $(5.0 \mathrm{~mol} \%)$; 用 $\mathrm{AlPW}_{12} \mathrm{O}_{40}$ 作催 化剂, 需要较长反应时间. 本文报道对空气稳定的全氟 辛基磺茂酸锆配合物催化吲哚与羰基化合物反应合成 二吲哚甲烷.

*E-mail: xhx1581@yahoo.com.cn

Received July 12, 2012; revised August 6, 2012; published online August 10, 2012.

Project supported by the National Natural Science Foundation of China (Nos. 21172061, 21273068).

国家自然科学基金(Nos. 21172061, 21273068)资助项目. 


\section{1 结果与讨论}

首先以吲哚与对羟基苯甲醛的反应作为模型进行 条件的探索. 实验表明, 在室温、四氢呋喃(THF)中, 催 化剂二(全氟辛基磺酸)二茂锆的量为 $1.0 \mathrm{~mol} \%$ 时, 吲哚 (2.0 mmol)与对羟基苯甲醛 $(1.0 \mathrm{mmol})$ 反应 $15 \mathrm{~min}$, 几乎 得到定量的二吲哚对差茎基甲烷. 在此条件下, 考察了 其他醛、酮与吲哚反应(Eq. 1), 结果见表 1.

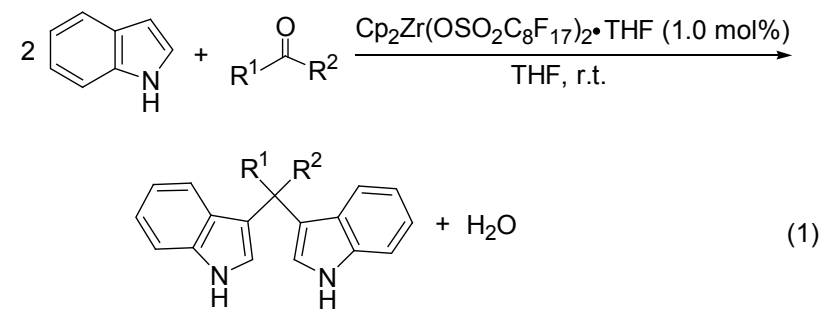

表 $1 \mathrm{Cp}_{2} \mathrm{Zr}\left(\mathrm{OSO}_{2} \mathrm{C}_{8} \mathrm{~F}_{17}\right)_{2} \cdot \mathrm{THF}$ 催化二吲哚甲烷衍生物的合成 Table 1 Synthesis of bis-indolylmethanes derivatives catalyzed by $\mathrm{Cp}_{2} \mathrm{Zr}\left(\mathrm{OSO}_{2} \mathrm{C}_{8} \mathrm{~F}_{17}\right)_{2} \cdot \mathrm{THF}$

\begin{tabular}{|c|c|c|c|c|}
\hline Compd. & $\mathrm{R}^{1}$ & $\mathrm{R}^{2}$ & Time/min & Yield $^{a} / \%$ \\
\hline $2 a$ & $\mathrm{Ph}$ & $\mathrm{H}$ & 10 & 99 \\
\hline $2 b$ & $m-\mathrm{NO}_{2} \mathrm{C}_{6} \mathrm{H}_{4}$ & $\mathrm{H}$ & 20 & 98 \\
\hline $2 \mathrm{c}$ & $p-\mathrm{HOC}_{6} \mathrm{H}_{4}$ & $\mathrm{H}$ & 15 & 92 \\
\hline 2d & $o-\mathrm{CF}_{3} \mathrm{C}_{6} \mathrm{H}_{4}$ & $\mathrm{H}$ & 18 & 89 \\
\hline $2 \mathrm{e}$ & $p-\mathrm{CF}_{3} \mathrm{C}_{6} \mathrm{H}_{4}$ & $\mathrm{H}$ & 12 & 99 \\
\hline $2 f$ & $p-\mathrm{BrC}_{6} \mathrm{H}_{4}$ & $\mathrm{H}$ & 16 & 87 \\
\hline $2 \mathrm{~g}$ & $p-\mathrm{ClC}_{6} \mathrm{H}_{4}$ & $\mathrm{H}$ & 25 & 86 \\
\hline $2 \mathrm{~h}$ & $p-\mathrm{CH}_{3} \mathrm{C}_{6} \mathrm{H}_{4}$ & $\mathrm{H}$ & 20 & 90 \\
\hline $2 \mathbf{i}$ & $\mathrm{CH}_{3} \mathrm{CH}_{2} \mathrm{CH}_{2}$ & $\mathrm{H}$ & 19 & 96 \\
\hline $2 \mathbf{j}$ & $\mathrm{Ph}$ & $\mathrm{CH}_{3}$ & 1500 & 94 \\
\hline $2 \mathrm{k}$ & & $\mathrm{H}$ & 960 & 98 \\
\hline
\end{tabular}

${ }^{a}$ Isolated yields.

由表 1 知, 芳香醛、芳香酮、脂肪醛、脂肪酮均能 与吲哚发生烷基化反应, 高收率形成二吲哚甲烷衍生 物; 苯环上取代基对反应没有明显影响. 相对于醛, 酮 需要较长的反应时间.

以吲哚与对羟基苯甲醛为模型, 对催化剂的重复使 用进行探究, 结果见表 2 .

表 2 催化剂二(全氟辛基磺酸)二茂锆的重复使用

Table 2 Recycle of catalyst $\left.\mathrm{Cp}_{2} \mathrm{Zr}\left(\mathrm{OSO}_{2} \mathrm{C}_{8} \mathrm{~F}_{17}\right)\right)_{2}$ THF

\begin{tabular}{ccccccc}
\hline Number & 1 & 2 & 3 & 4 & 5 & 6 \\
\hline Yield $^{a} \%$ & 98 & 98 & 96 & 94 & 93 & 91 \\
\hline${ }^{a}$ Iso
\end{tabular}

${ }^{a}$ Isolated yields.

实验结果表明, 二 (全氟辛基磺酸) 二茂锆 $\left[\mathrm{Cp}_{2} \mathrm{Zr}\left(\mathrm{OSO}_{2} \mathrm{C}_{8} \mathrm{~F}_{17}\right)_{2} \cdot \mathrm{THF}\right]$ 重复催化使用 6 次后, 催化产 率仍能达到 $91 \%$.

\section{2 结论}

二(全氟辛基磺酸)二茂锆在室温、THF 中就能高效 的催化吲哚与醛、酮反应生成二吲哚甲烷衍生物, 且配 合物能重复使用，催化剂具有耐水、耐氧的特性，反应 不需要在严格的无水条件下进行, 而且产物收率高, 易 于分离、纯化，此方法为二吲哚甲烷衍生物制备提供一 条新的有效途径.

\section{3 实验部分}

\section{1 仪器与试剂}

${ }^{1} \mathrm{H} \mathrm{NMR},{ }^{13} \mathrm{C} \mathrm{NMR}$ (以 $\mathrm{CDCl}_{3}$ 作溶剂, $\mathrm{TMS}$ 为内标) 用 BRUKER AC-P400 型仪测定, 质谱由 VG Auto Spec-300 仪测定; 硅胶为青岛海洋化工厂产品; 溶剂 $\mathrm{THF}$ 未进行除水处理. 吲哚、醛、酮均在 Aldrich 公司 购买, 其他试剂均为分析纯，二茂全氟辛基磺酸锆按文 献方法制备 ${ }^{[12]}$.

\section{2 二茂全氟辛基磺酸锆催化吲哚与羰基化合物反 应}

在 $50 \mathrm{~mL}$ 圆底烧瓶中, 依次加入羰基化合物(1.0 $\mathrm{mmol})$, THF (2.0 mL), 吲哚 $(0.234 \mathrm{~g}, 2.0 \mathrm{mmol})$, 二(全氟 辛基磺酸)二茂锆 $(0.012 \mathrm{~g}, 0.01 \mathrm{mmol})$, 室温下搅拌反 应, TLC 跟踪反应. 反应完成后，旋干溶剂，乙醚溶解稀 释, 过滤分离回收催化剂, 滤液旋干得到粗产品, 柱层 析分离 $\left(V_{\text {石油瞵 }}: V_{\text {二氯甲烷 }}=8: 1\right)$ 得到目标产物.

\section{3 化合物光谱数据}

3,3'-二吲哚基苯甲烷(2a): 浅粉红色固体, m.p. $127 \sim 129{ }^{\circ} \mathrm{C}$ (文献值 $\left.{ }^{[19]} 125 \sim 127{ }^{\circ} \mathrm{C}\right) ;{ }^{1} \mathrm{H}$ NMR $(400$ $\left.\mathrm{MHz}, \mathrm{CDCl}_{3}\right) \delta: 5.81(\mathrm{~s}, 1 \mathrm{H}), 6.61(\mathrm{~d}, J=1.6 \mathrm{~Hz}, 2 \mathrm{H})$, $6.99(\mathrm{t}, J=7.8 \mathrm{~Hz}, 2 \mathrm{H}), 7.14 \sim 7.22(\mathrm{~m}, 3 \mathrm{H}), 7.28(\mathrm{~d}, J=$ $6.8 \mathrm{~Hz}, 2 \mathrm{H}), 7.32 \sim 7.35(\mathrm{~m}, 4 \mathrm{H}), 7.38(\mathrm{~d}, J=8.4 \mathrm{~Hz}, 2 \mathrm{H})$, 7.83 (s, 2H); ${ }^{13} \mathrm{C}$ NMR (100 MHz, $\left.\mathrm{CDCl}_{3}\right) \delta: 144.3,136.7$, $128.8,128.5,127.3,126.4,123.7,122.2,120.0,119.7$, 119.3, 111.3, 40.2; MS (EI) $m / z: 322\left(\mathrm{M}^{+}\right)$.

3,3'-二吲哚基-3-硝基苯基甲烷(2b): 浅粉红色固体, m.p. 261 264 ${ }^{\circ} \mathrm{C}$ (文献值 ${ }^{[19]} 260 \sim 262{ }^{\circ} \mathrm{C}$ ); ${ }^{1} \mathrm{H}$ NMR (400 $\left.\mathrm{MHz}, \mathrm{CDCl}_{3}\right) \delta: 5.99(\mathrm{~s}, 1 \mathrm{H}), 6.65(\mathrm{~s}, 2 \mathrm{H}), 7.02(\mathrm{t}, J=8.2$ $\mathrm{Hz}, 2 \mathrm{H}), 7.18 \sim 7.25(\mathrm{~m}, 2 \mathrm{H}), 7.34 \sim 7.38(\mathrm{~m}, 4 \mathrm{H}), 7.43(\mathrm{t}$, $J=8.0 \mathrm{~Hz}, 1 \mathrm{H}), 7.69$ (d, $J=7.6 \mathrm{~Hz}, 1 \mathrm{H}), 7.99$ (s, 1H), 8.08 $(\mathrm{d}, J=10 \mathrm{~Hz}, 1 \mathrm{H}), 8.21(\mathrm{~s}, 1 \mathrm{H}) ;{ }^{13} \mathrm{C} \mathrm{NMR}(100 \mathrm{MHz}$, $\left.\mathrm{CDCl}_{3}\right) \delta: 147.8,139.0,136.5,135.1,129.5,127.4,125.4$, $123.0,121.7,120.9,119.8,118.8,112.1,111.1,53.6$; MS (EI) $m / z: 367\left(\mathrm{M}^{+}\right)$.

3,3'-二吲哚基-4-差基苯基甲烷(2c): 浅粉红色固体, m.p. $126 \sim 127{ }^{\circ} \mathrm{C}$ (文献值 $\left.{ }^{[19]} 122 \sim 126{ }^{\circ} \mathrm{C}\right) ;{ }^{1} \mathrm{H}$ NMR $(400$ 
$\left.\mathrm{MHz}, \mathrm{CDCl}_{3}\right) \delta: 6.00(\mathrm{~s}, 1 \mathrm{H}), 6.74(\mathrm{~d}, J=1.6 \mathrm{~Hz}, 2 \mathrm{H})$, $6.84 \sim 6.86(\mathrm{~m}, 2 \mathrm{H}), 7.02(\mathrm{t}, J=7.0 \mathrm{~Hz}, 2 \mathrm{H}), 7.17 \sim 7.20$ (m, 4H), 7.34 7.40 (m, 4H), $8.00(\mathrm{~s}, 2 \mathrm{H}) ;{ }^{13} \mathrm{C}$ NMR $(100$ $\left.\mathrm{MHz}, \mathrm{CDCl}_{3}\right) \delta: 155.5,136.5,130.7,130.4,127.4,123.0$, $121.7,119.8,118.8,115.8,112.1,111.1,54.6$; MS (EI) $m / z: 338\left(\mathrm{M}^{+}\right)$.

3,3'-二吲哚基-2-三氟甲基苯基甲烷(2d): 浅粉红色 固体，m.p. $223 \sim 225{ }^{\circ} \mathrm{C}$ (文献值 ${ }^{[20]} 224 \sim 226{ }^{\circ} \mathrm{C}$ ); ${ }^{1} \mathrm{H}$ NMR (400 MHz, $\left.\mathrm{CDCl}_{3}\right) \delta: 6.30(\mathrm{~s}, 1 \mathrm{H}), 6.56(\mathrm{~d}, J=1.6$ $\mathrm{Hz}, 2 \mathrm{H}), 7.01(\mathrm{t}, J=7.0 \mathrm{~Hz}, 2 \mathrm{H}), 7.17(\mathrm{t}, J=7.2 \mathrm{~Hz}, 2 \mathrm{H})$, $7.32 \sim 7.41(\mathrm{~m}, 6 \mathrm{H}), 7.47$ (d, $J=7.2 \mathrm{~Hz}, 1 \mathrm{H}), 7.73$ (d, $J=$ $7.2 \mathrm{~Hz}, 1 \mathrm{H}), 7.91$ (s, 2H); ${ }^{13} \mathrm{C}$ NMR (100 MHz, $\left.\mathrm{CDCl}_{3}\right) \delta$ : $137.0,136.5,131.9,130.9,127.8,127.4,126.1,126.0$, 124.0, 123.0, 121.7, 119.8, 118.8, 112.1, 111.1, 52.1; MS (EI) $m / z: 390\left(\mathbf{M}^{+}\right)$.

3,3'二二吲哚基-4-三氟甲基苯基甲烷(2e): 浅粉红色 固体, m.p. 226 227 ${ }^{\circ} \mathrm{C}$ (文献值 ${ }^{[20]} 229 \sim 230{ }^{\circ} \mathrm{C}$ ); ${ }^{1} \mathrm{H}$ NMR (400 MHz, $\left.\mathrm{CDCl}_{3}\right) \delta: 5.93$ (s, 1H), 6.61 (d, $J=1.6$ $\mathrm{Hz}, 2 \mathrm{H}), 7.01$ (t, $J=7.8 \mathrm{~Hz}, 2 \mathrm{H}), 7.18$ (t, $J=7.8 \mathrm{~Hz} 2 \mathrm{H}$ ), 7.35 (d, $J=8.8 \mathrm{~Hz}, 4 \mathrm{H}), 7.44$ (d, $J=8.4 \mathrm{~Hz}, 2 \mathrm{H}), 7.52$ (d, $J=8.0 \mathrm{~Hz}, 2 \mathrm{H}), 7.91(\mathrm{~s}, 2 \mathrm{H}) ;{ }^{13} \mathrm{C} \mathrm{NMR}\left(100 \mathrm{MHz}, \mathrm{CDCl}_{3}\right)$ $\delta: 141.4,136.5,129.3,128.0,127.4,125.0,124.1,123.0$, 121.7, 119.8, 118.8, 112.1, 111.1, 54.6; MS (EI) $\mathrm{m} / z: 390$ $\left(\mathrm{M}^{+}\right)$.

3,3'-二吲哚基-4-溴代苯基甲烷(2f): 浅粉红色固体, m.p. 101 103 ${ }^{\circ} \mathrm{C}$ (文献值 ${ }^{[21]} 100 \sim 101{ }^{\circ} \mathrm{C}$ ) $;{ }^{1} \mathrm{H}$ NMR $(400$ $\left.\mathrm{MHz}, \mathrm{CDCl}_{3}\right) \delta: 5.84(\mathrm{~s}, 1 \mathrm{H}), 6.65(\mathrm{~d}, J=1.6 \mathrm{~Hz}, 2 \mathrm{H})$, $7.01(\mathrm{t}, J=7.6 \mathrm{~Hz}, 2 \mathrm{H}), 7.16 \sim 7.20(\mathrm{~m}, 4 \mathrm{H}), 7.35 \sim 7.40$ $(\mathrm{m}, 6 \mathrm{H}), 7.93(\mathrm{~s}, 2 \mathrm{H}) ;{ }^{13} \mathrm{C}$ NMR $\left(100 \mathrm{MHz}, \mathrm{CDCl}_{3}\right) \delta$ : $137.1,136.5,131.5,131.2,127.4,123.0,121.7,120.1$, 119.8, 118.8, 112.1, 111.1, 54.6; MS (EI) $m / z: 400\left(\mathrm{M}^{+}\right)$.

3,3'-二吲哚基-4-氯代苯基甲烷(2g): 浅粉红色固体, m.p. 102 106 ${ }^{\circ} \mathrm{C}$ (文献值 ${ }^{[19]} 103 \sim 105{ }^{\circ} \mathrm{C}$ ) $;{ }^{1} \mathrm{H}$ NMR $(400$ $\left.\mathrm{MHz}, \mathrm{CDCl}_{3}\right) \delta: 5.86(\mathrm{~s}, 1 \mathrm{H}), 6.65(\mathrm{~d}, J=1.2 \mathrm{~Hz}, 2 \mathrm{H})$, $7.01(\mathrm{t}, J=7.8 \mathrm{~Hz}, 2 \mathrm{H}), 7.16 \sim 7.28(\mathrm{~m}, 5 \mathrm{H}), 7.35 \sim 7.37$ (m, 4H), 7.93 (s, 2H); ${ }^{13} \mathrm{C}$ NMR (100 MHz, $\left.\mathrm{CDCl}_{3}\right) \delta$ : 142.6, 136.2, 131.3, 130.1, 128.1, 126.6, 123.1, 122.1, 119.8, 119.4, 119.2, 111.1, 39.6; MS (EI) $m / z: 356.5\left(\mathrm{M}^{+}\right)$.

3,3'-二吲哚基-4-甲基苯基甲烷(2h): 浅粉红色固体, m.p. 97 98 ${ }^{\circ} \mathrm{C}$ (文献值 ${ }^{[19]} 95 \sim 98{ }^{\circ} \mathrm{C}$ ); ${ }^{1} \mathrm{H}$ NMR $(400$ $\left.\mathrm{MHz}, \mathrm{CDCl}_{3}\right) \delta: 2.32$ (s, 3H), $5.85(\mathrm{~s}, 1 \mathrm{H}), 6.67$ (t, $J=1.0$ $\mathrm{Hz}, 2 \mathrm{H}), 6.99$ (t, $J=7.6 \mathrm{~Hz}, 2 \mathrm{H}), 7.08(\mathrm{~d}, J=8.0 \mathrm{~Hz}, 2 \mathrm{H})$, $7.16(\mathrm{t}, J=7.6 \mathrm{~Hz}, 2 \mathrm{H}), 7.23(\mathrm{~d}, J=8.0 \mathrm{~Hz}, 2 \mathrm{H}), 7.35$ (d, $J=7.6 \mathrm{~Hz}, 2 \mathrm{H}), 7.39$ (d, $J=7.6 \mathrm{~Hz}, 2 \mathrm{H}), 7.90(\mathrm{~s}, 2 \mathrm{H}) ;{ }^{13} \mathrm{C}$ NMR $\left(100 \mathrm{MHz}, \mathrm{CDCl}_{3}\right) \delta: 141.5,136.4,135.1,129.3$,
$128.5,127.3,123.4,122.5,120.8,119.8,119.1,111.1$, 40.6, 21.3; MS (EI) $m / z: 336\left(\mathrm{M}^{+}\right)$.

3,3'-二吲哚基丁烷 (2i): 白色固体, m.p. 63 64 ${ }^{\circ} \mathrm{C}$ (文献值 $\left.{ }^{[22]} 65 \sim 67{ }^{\circ} \mathrm{C}\right) ;{ }^{1} \mathrm{H}$ NMR $\left(400 \mathrm{MHz}, \mathrm{CDCl}_{3}\right) \delta$ : $0.92 \sim 0.96(\mathrm{~m}, 4 \mathrm{H}), 1.39 \sim 1.45(\mathrm{~m}, 4 \mathrm{H}), 2.15 \sim 2.21(\mathrm{~m}$, $2 \mathrm{H}), 4.48(\mathrm{t}, J=7.4 \mathrm{~Hz}, 1 \mathrm{H}), 6.94(\mathrm{~d}, J=2.4 \mathrm{~Hz}, 2 \mathrm{H}), 7.02$ (t, $J=8.2 \mathrm{~Hz}, 2 \mathrm{H}), 7.11 \sim 7.15(\mathrm{~m}, 2 \mathrm{H}), 7.30$ (d, $J=7.6$ $\mathrm{Hz}, 2 \mathrm{H}), 7.59$ (d, $J=7.6 \mathrm{~Hz}, 2 \mathrm{H}), 7.89$ (s, 2H); ${ }^{13} \mathrm{C} \mathrm{NMR}$ $\left(100 \mathrm{MHz}, \mathrm{CDCl}_{3}\right) \delta: 139.8,136.5,124.2,121.7,119.8$, $118.8,115.4,111.1,49.4,43.0,19.7,14.4$; MS (EI) $\mathrm{m} / \mathrm{z}$ : $288\left(\mathrm{M}^{+}\right)$.

1,1'-(3,3'-二吲哚基-苯基)乙烷(2j): 白色固体, m.p. $136 \sim 138{ }^{\circ} \mathrm{C}$ (文献值 ${ }^{[22]} 135 \sim 137{ }^{\circ} \mathrm{C}$ ); ${ }^{1} \mathrm{H}$ NMR $(400$ $\left.\mathrm{MHz}, \mathrm{CDCl}_{3}\right) \delta: 2.35(\mathrm{~s}, 3 \mathrm{H}), 6.59$ (d, $\left.J=2.8 \mathrm{~Hz}, 2 \mathrm{H}\right)$, $6.92(\mathrm{t}, J=7.1 \mathrm{~Hz}, 2 \mathrm{H}), 7.12(\mathrm{t}, J=7.1 \mathrm{~Hz}, 2 \mathrm{H}), 7.18 \sim$ $7.25(\mathrm{~m}, 4 \mathrm{H}), 7.30 \sim 7.33(\mathrm{~m}, 4 \mathrm{H}), 7.39(\mathrm{~d}, J=7.2 \mathrm{~Hz}$, 2H), $7.81(\mathrm{~s}, 2 \mathrm{H}) ;{ }^{13} \mathrm{C}$ NMR $\left(100 \mathrm{MHz}, \mathrm{CDCl}_{3}\right) \delta: 140.8$, $136.5,130.7,128.6,127.4,125.9,123.0,121.7,119.8$, 118.8, 112.1, 111.1, 50.6, 15.0; MS (EI) $m / z: 336\left(\mathrm{M}^{+}\right)$.

3,3'-二吲哚基环已烷(2k): 白色固体, m.p. 86 87 ${ }^{\circ} \mathrm{C}\left(\right.$ 文献值 $\left.{ }^{[22]} 89 \sim 91{ }^{\circ} \mathrm{C}\right) ;{ }^{1} \mathrm{H}$ NMR $\left(400 \mathrm{MHz}, \mathrm{CDCl}_{3}\right) \delta$ : $5.57(\mathrm{t}, J=2.2 \mathrm{~Hz}, 2 \mathrm{H}), 1.59 \sim 1.64(\mathrm{~m}, 4 \mathrm{H}), 2.53$ (t, $J=$ $5.8 \mathrm{~Hz}, 4 \mathrm{H}), 6.88(\mathrm{t}, J=5.6 \mathrm{~Hz}, 2 \mathrm{H}), 7.03 \sim 7.06(\mathrm{~m}, 4 \mathrm{H})$, $7.24(\mathrm{t}, J=9.2 \mathrm{~Hz}, 2 \mathrm{H}), 7.54(\mathrm{~d}, J=4.0 \mathrm{~Hz}, 2 \mathrm{H}), 7.82(\mathrm{~s}$, $2 \mathrm{H}) ;{ }^{13} \mathrm{C}$ NMR $\left(100 \mathrm{MHz}, \mathrm{CDCl}_{3}\right) \delta: 210.8,139.8,136.5$, 124.2, 121.7, 119.8, 118.8, 115.4, 111.1, 53.1, 46.0, 41.3, 28.2; MS (EI) $m / z: 314\left(\mathrm{M}^{+}\right)$.

\section{References}

[1] Yamamoto, H. Lewis Acids in Organic Synthesis, Wiley-VCH, Weinheim, Germany, 2000.

[2] Ye, J.-H.; Xu, G.-J.; Zhang, W.-C.; Wang, S.-B. Chin. J. Org. Chem. 2009, 29, 1664 (in Chinese). (叶家海, 徐国际, 张文超, 田桂蓉, 有机化学, 2009, 29, 1664.)

[3] Wu, J.-Y.; Fang, H.; Xu, W.-F. Chin. J. Org. Chem. 2009, 29, 1175 (in Chinese) (吴记勇, 芳浩, 徐文方, 有机化学, 2009, 29, 1175.)

[4] Zhao, Y.; Ye, C.-C.; Tan, X..-Y.; Yang, Z. Chin. J. Org. Chem. 2009 29, 643 (in Chinese). (赵莹, 叶翠层, 谭晓叶, 扬志, 有机化学, 2009, 29, 643.)

[5] Hollis, T. K.; Robison, N. P.; Bosnich, B. Tetrahedron Lett. 1992, 33,6423 .

[6] Hollis, T. K.; Bosnich, B. J. Am. Chem. Soc. 1995, 117, 4570.

[7] Ohmori, K.; Hatakeyama, K.; Ohrui, H.; Suzuki, K. Tetrahedron 2004, 60, 1365.

[8] Hollis, T. K.; Robinson, N. P.; Bosnich, B. Organometallics 1992, 11,2745

[9] An, D.; Peng, Z.; Orita, A.; Kurita, A.; Man, S.; Ohkubo, K.; Li, X.; Fukuzumi, S.; Otera, J. Chem. Eur. J. 2006, 12, 1642.

[10] Qiu, R. H.; Xu, X. H.; Li, Y. H.; Zhang, G. P.; Shao, L. L.; An, D. L.; Yin, S. F. Chem. Commun. 2009, 1679. 
[11] Qiu, R. H.; Zhang, G. P.; Zhu, Y. Y.; Xu, X. H.; Shao, L. L.; Li, Y. H.; An, D. L.; Yin, S. F. Chem. Eur. J. 2009, 15, 6488.

[12] Qiu, R. H.; Zhang, G. P.; Xu, X. H.; Zou, K. B.; Shao, L. L.; Fang, D. W.; Li, Y. H.; Orita, A.; Saijo, R.; Mineyama, H.; Suenobu, T.; Fukuzumi, S.; An, D. L.; Otera, J. J. Organomet. Chem. 2009, 1524.

[13] Qiu, R. H.; Zhu, Y. Y.; Xu, X. H.; Li, Y. H.; Shao, L. L.; Ren, X. F.; Cai, X. T.; An, D. L.; Yin, S. F. Catal. Commun. 2009, 10, 1889.

[14] Qiu, R. H.; Yin, S. F.; Zhang, X. W.; Xia, J.; Xu, X. H.; Luo, S. L. Chem. Commun. 2009, 4759.

[15] Qiu, R. H.; Zhang, G. P.; Xu, X. H.; Peng, L. F.; Zhao, Y. L.; Li, N. B.; Yin, S. F. Chem. Eur. J. 2012, 18, 6172.

[16] Mi, X. L.; Luo, S. Z.; He, J. Q. Tetrahedron Lett. 2004, 45, 4567.
[17] Firouzabadi, H.; Iranpoor, N.; Jafari, A. A. A. J. Mol. Catal. A: Chem. 2006, 244, 168.

[18] Firouzabadi, H.; Iranpoor, N.; Jafarpour, M. J. Mol. Catal. A: Chem. 2006, 253, 249.

[19] Aliyan, H.; Fazaeli, R.; Naghash, H. J. Heteroat. Chem. 2009, 20, 325.

[20] Ma, Z. H.; Han, H. B.; Zhou, Z. B. J. Mol. Catal. A: Chem. 2009, $311,46$.

[21] Khaksar, S.; Ostad, S. M. J. Fluorine Chem. 2011, 132, 937.

[22] Lv, W.-H.; Liu, X.-H.; Liu, D.-Y. Chin. J. Catal. 2009, 30, 1287 (in Chinese).

(吕文辉, 刘兴海, 刘冬妍, 催化学报, 2009, 30, 1287.) 